\title{
Aleuromantie \\ VON DER aLtorientalischen KunSt, MIT HiLfE VON OPFERMEHL DAS MASS GÖTTLICHEN WOHLWOLLENS ZU ERMITTELN
}

\author{
Stefan M. Maul
}

Ein an den Sonnengott Schamasch gerichtetes Gebet, welches uns in einer Niederschrift aus der neuassyrischen Zeit überliefert ist, zeigt, daß jedem einzelnen Menschen stets die Möglichkeit offenstehen sollte, durch regelmäßige Opfergaben eine stabile Beziehung zu den über ihm stehenden Mächten aufzubauen, um so von einer ihm wohlgesonnenen Gottheit beständig Schutz und Zuwendung zu erfahren. Der materielle Wert der Opfergaben galt als zweitrangig. Denn jeder sollte nach Maßgabe der eigenen Vermögensverhältnisse zur Versorgung der Götter beitragen können. In dem Gebet heißt es nämlich:

Der Opferschauer bringt dir Zeder (als Räucherwerk), die Witwe Röstmehl, die arme Frau Öl, der Reiche in seinem Reichtum ein Lamm. ${ }^{1}$

Von solchen Gaben konnte der mesopotamische Opferspender freilich weit mehr erwarten als nur die diffuse Hoffnung, sich damit die bedachte Gottheit gewogen zu machen. Denn in Quellen aus allen historischen Perioden des Alten Zweistromlandes treffen wir auf die uns zunächst ganz eigentümlich anmutende Überzeugung, daß sich die durch die Gabe des Opfers erhoffte göttliche Zuwendung unverzüglich in dem Geopferten selbst zu zeigen beginne. Man glaubte nämlich, an und in einem Opfertier, nicht zuletzt in dessen Eingeweiden, vor allem

1 Butler 1998, 274:21. Ganz ähnliche Regeln sind z. B. in Lev 5:7ff. beschrieben. 
aber auf der Leber Zeichen göttlichen Wohlwollens ausmachen, ja das Maß der Gunst ablesen zu können, die die Götter dem Opferspender gewährten. Über den im tiefen Dunkel der Vorgeschichte liegenden Ursprung dieses uralten, zumeist „Opferschau“ genannten Verfahrens wissen wir nichts. Zweifelsohne wird er aber in dem Wunsch zu suchen sein, anhand sichtbarer Zeichen zu überprüfen, ob das dargebrachte Opfer bei den Göttern Wohlgefallen finde. ${ }^{2}$ Die sachkundige, sorgfältige Inspektion der Gabe eines Opfertiers eröffnete so jedem einzelnen Menschen nicht allein die Möglichkeit, jederzeit in eine regelrechte Kommunikation mit den Göttern einzutreten, sondern gestattete auch, von dem diagnostizierten Ausmaß göttlichen Wohlwollens auf die eigenen Aussichten auf Erfolg und Gelingen zurückzuschließen. Dabei lag nahe, eine solche Prüfung insbesondere dann vorzunehmen, wenn es wichtige Entscheidungen zu fällen galt. Denn diese ließen sich mit weit größerer Zuversicht treffen, wenn zuvor die schicksalsmächtigen Götter selbst in dem ihnen Dargebrachten kundgetan hatten, daß sie dem Opferspender auch weiterhin ihre Gunst erweisen würden. Es war daher nur ein kleiner Schritt, die Inspektion eines Opfertiers mit einer konkreten, an die Götter gerichteten Anfrage zu verbinden und in dem Befund der Opferschau göttliche Befürwortung und Ablehnung einer Absicht oder eines Plans zu suchen. In der Mitte des dritten vorchristlichen Jahrtausends war dieser Schritt schon lange vollzogen. Denn Keilschriftquellen belegen, daß die Opferschau bereits zu dieser Zeit von Königen und Fürsten Mesopotamiens und Syriens als ein Orakelverfahren genutzt wurde, mit dessen Hilfe Entscheidungen mit der Autorität eines göttlichen placet versehen wurden. ${ }^{3}$

Dennoch war mit dem Gedanken der Opferschau keineswegs zwingend die Vorstellung verbunden, daß als Preis für eine göttliche „Weisung“ (têrtum) eine Kreatur ihr Leben lassen müsse. Über Jahrtausende hinweg wurden nämlich Orakelentscheide auch aus jenen bescheideneren Opfergaben herausgelesen, die pflanzlichen Ursprungs waren: aus Mehl, Öl und Räucherwerk. Obgleich man in dem Leben eines Opfertiers wohl tatsächlich einen den Göttern übereigneten

2 Der Zusammenhang der zwischen der Prüfung, ob ein Tieropfer als von der Gottheit angenommen betrachtet werden kann, und der Möglichkeit besteht, den Opferschaubefund für ein Orakel zu nutzen, wird in dem altbabylonischen Brief Durand 1988, 258f. Text Nr. 92 sehr deutlich.

3 Vgl. Maul 2003, 70f. mit weiterführender Literatur. 
Ersatz sah für das als bedroht empfundene Leben des opfernden und um „Weisung“ bittenden Menschen, kann das Töten somit nicht als ein Kernstück des Opferschaugedankens gelten. Dennoch scheint es so, als sei das Vergießen von Blut im Rahmen einer Opferschau immer dann als notwendig, zumindest aber als ratsam erachtet worden, wenn die Opferschau den Ausweg aus einer Situation „weisen“ sollte, die man als existenzbedrohend empfand. Denn, wenn es wirklich ernst wurde, bevorzugte man in allen Perioden der altorientalischen Geschichte - sofern es die gegebenen Verhältnisse nur zuließen - die an einem Tier vollzogene Opferschau; wohl nicht nur, weil man sich von diesem Verfahren die präziseste Auskunft erhoffte, sondern eben auch, weil das Opfertier an des Menschen Stelle sterben sollte. ${ }^{4}$ Freilich mußten die Ärmeren immer mit „Weisungen“ Vorlieb nehmen, die die Opferschauer aus ihren bescheidenen Gaben lasen.

Altorientalische Mythen ${ }^{5}$ geben allerdings deutlich zu erkennen, daß der Art der Opfergabe weit weniger Bedeutung beigemessen wurde, als der Arbeit, die Menschen investieren mußten, um der Natur die den Göttern dargebrachten Gaben abzugewinnen. Und so scheint es, daß man die Gnade der Gottheit, in der Opferschau dem bittenden Menschen eine „Weisung“ zu erteilen, vor allem als eine Leistung ansah, die die Gottheit als Gegengabe für die Frucht menschlicher Arbeit zu erbringen gewillt war, ganz unabhängig davon, ob die Gabe pflanzlichen oder tierischen Ursprungs war. Es ist jedenfalls auffällig, daß für die Opferschau nur die in harter Arbeit zu gewinnenden Erzeugnisse von Land- und Viehwirtschaft Verwendung fanden. Wir kennen keine altorientalische Opferschauverfahren, die etwa mit Wildtieren ${ }^{6}$ oder mit in der freien Natur gesammelten Gütern vollzogen werden konnten. Die für das Orakelverfahren

4 Vgl. Blome 1934, 48ff. Auch in medizinisch-therapeutischen und kathartischen Verfahren ist der Gedanke häufig anzutreffen, daß eine Krankheit oder ein Unheil auf ein Tier übergehen soll, das als Substitut für den erkrankten Menschen getötet wurde (vgl. z. B. Schramm 2008, 40-43:1-44).

5 Dazu ausführlich Maul 2007, 129-136 und ders., 2008, 76-78.

6 In der ethnologischen Fachliteratur finden sich bemerkenswerte Parallelen. Noch heute wird von den Tishana-Me'en in Südwestäthiopien Eingeweideschau betrieben, aber ausschließlich mit domestizierten Tieren, da nach Ansicht der Zeichendeuter allein diese ,part of the social as well as of the natural world" seien und so als „,mediators" between these two worlds" und als „,vehicles of meaning“ dienen können (Abbink 1993, 709). 
verwendeten Lämmer waren wohl ebenso wie die taubenartigen Vögel ${ }^{7}$ Haustiere, die mühsam aufgezogen werden mußten, und auch Mehl, Öl und Räucherwerk sind das Ergebnis aufwendiger, mit harter Arbeit verbundener Herstellungsprozesse.

Im Auftrag der Mächtigen und Reichen wurde das Urteil der Götter wohl nur dann mit Hilfe von Mehl, Öl und Räucherwerk eingeholt, wenn es galt, einen Orakelentscheid in besonders kurzer Zeit herbeizuführen. Denn die Zeichen, die Mehl, Öl und Räucherwerk liefern, ließen sich nicht nur recht kostengünstig generieren, sondern man konnte sie auch - ganz anders als in den aufwendigen Verfahren der Inspektion von Lamm und Vogel - in großer Schnelle herbeiführen und deuten. Die ,kleinen' Opferschauverfahren erwiesen sich daher auch als geeignet, um sich im Rahmen anderer, mit größerem Aufwand betriebener Divinationsprozeduren in bestimmten Augenblicken des göttlichen Wohlwollens zu versichern ${ }^{8}$ und um - wie dies wohl durchaus üblich war - die Richtigkeit der Ergebnisse, die mit einer Eingeweideschau erzielt worden waren, zu überprüfen.

\section{Die mit der Gabe von Mehl verbundene Opferschau}

Der Stellenwert, den in Mesopotamien das Getreide für die Speisung der Götter besaß, entspricht der großen Bedeutung, die dem Getreide auch für die Ernährung der Menschen zukam. Nur in aufbereiteter Form, als Mehl, Brot oder in Gestalt verschiedener Gerichte, wurde es auf die Opfertische der Götter gelegt und auf diese Weise dargebracht. Eine Gabe von Mehl konnte freilich auch mit Harzen, Hölzern, Kräutern und anderen Duftstoffen vermischt als Räucherwerk Verwendung finden und in Gestalt des aufsteigenden Rauches die Götter erreichen. ${ }^{9}$

7 Zu der mit Geflügel betriebenen Opferschau siehe Nougayrol 1967; Labat 1974, 158-176 (Text Nr. 7); Archi 1975, 139-141; Tsukimoto 1982; Starr 1983, 60-63; Durand 1997; Maul 2003, 82f.; Edzard 2004, 545f. und Haas 2008, 103ff. Ein Hinweis darauf, daß die taubenartigen Vögel, zu denen wohl auch der ișșū hurri zählte (so Durand 1988, 38 und Durand 1997, 274), aufgezogene Haus- und keine Wildtiere waren, findet sich in Starr 1983, 62:17. Dort ist gesagt, daß der für eine Opferschau verwendete Vogel ,vom Tisch des Gottes“ aß.

8 Vgl. z. B. Zimmern 1901, 104, Text Nr. 1-20:120-126.

9 Vgl. Maul 1994, 48ff. 
Bei Bedarf dürfte das zum Opfer bestimmte Mehl dem um eine „Weisung“ bittenden Opferspender - sei es in Gänze oder sei es nur zu einem Teil - als Medium für eine Orakelanfrage gedient haben.

Die keilschriftlichen Zeugnisse für das Verfahren, mit Hilfe von ausgestreutem Mehl einen Orakelbescheid zu generieren, sind freilich so dürftig, daß Einzelheiten vorerst völlig im Dunkeln bleiben müssen. Man darf zwar annehmen, daß die Mehlwahrsagung, so wie die übrigen durch Opferschau erstellten Orakel, am frühen Morgen bei Sonnenaufgang durchgeführt wurde. Wir müssen aber konstatieren, daß wir einfach nichts über die Umstände und die rituelle Einbindung dieses Divinationsverfahrens wissen, ja wir sind nicht einmal im Stande zu sagen, ob es ausschließlich von professionellen Experten ausgeübt wurde. Selbst über die Popularität des Orakels können wir mangels Quellen kaum mehr als Mutmaßungen anstellen. Denn das vergleichsweise simple Verfahren der Mehlwahrsagung, das sehr wahrscheinlich vor allem von den weniger Betuchten in Anspruch genommen wurde, interessierte die gelehrten Opferschauer nur am Rande, und die divinatorische Beratung der einfachen Leute fand ohnehin keinen Niederschlag in ihren schriftlichen Hinterlassenschaften.

So gibt es kaum mehr als eine einzige, von Schülerhand geschriebene Omensammlung aus der spätaltbabylonischen Zeit, die uns Einblick in die Kunst, aus Opfermehl Zeichen zu lesen, gewährt, und immerhin unter Beweis stellt, daß auch Verfahrensweisen und Hermeneutik dieser Divinationsform Gegenstand der Ausbildung professioneller Zeichendeuter sein konnten. ${ }^{10}$

Obgleich die Tafel nur 14 Omeneinträge enthält, dokumentiert sie zwei Methoden, einen Orakelbescheid zu erstellen.

Für die erste wurde eine Portion Mehl, unter die man eine Emmerähre ${ }^{11}$ gemischt hatte, in ein Gefäß gegeben und mit einem Mal auf ein Brett, ein Platte oder eine flache Schale geschüttet, um dann zu beobachten, wie sich Ähre und Mehl zueinander verhalten würden. Obgleich die Regeln dieses Verfahrens nirgendwo explizit formuliert wurden, reichen die auf der Schülertafel zusammengestellten, klug ausgewählten neun zugehörigen Omina aus, ${ }^{12}$ die im Grunde sehr

10 Edition der Omensammlung: Nougayrol 1963; vgl. die deutsche Übersetzung: Pientka-Hinz 2008, 29-31.

11 Gegen Maul 2003, 85 handelt es sich wohl nicht um Emmerkörner.

12 Nougayrol 1963, 382-384:1-24. 
einfachen, dem Verfahren zu Grunde liegenden Prinzipien zu erschlieBen. Sie erweisen sich als die Elementarregeln aller Opferschauen.

Die Emmerähre stand bei dem Orakelverfahren für den Fragesteller selbst, während das feine Mehl, welches die Ähre in dem einen Fall freigeben, in dem anderen ganz oder nur teilweise bedecken mochte, das Ausmaß der ,Verschüttung“ anzeigte, dem sich der Fragende ausgesetzt sehen würde. In gewissem Sinn entspricht damit die frei daliegende Emmerähre den zahlreichen positiv konnotierten Markierungen, die auf Leber und Eingeweiden beobachtet werden konnten, während die grundsätzlich negativ gewerteten Markierungen der Eingeweideschau in der Mehldivination ihr Analogon in der „Verschüttung“ der Ähre finden. ${ }^{13}$

Dennoch wurde die „Verschüttung“ der Ähre - ebenso wie die negativ konnotierten Markierungen auf den inspizierten Eingeweiden nicht immer negativ beurteilt. Ihre Wertung hing maßgeblich davon ab, an welcher Stelle der Unterlage, auf die man Mehl und Ähre geschüttet hatte, der Befund beobachtet wurde. Die Unterlage war nämlich, wie uns die Omenhandschrift lehrt, in zwei Bereiche aufgeteilt, die die Opferschauer ,Sonnenaufgang“ und „Sonnenuntergang“" nannten. Und man wird wohl in der Annahme nicht fehlgehen, daß die Zeichendeuter am frühen Morgen bei der Inspektion des ausgeschütteten Mehls die als „Sonnenaufgang“ bezeichnete Seite der Unterlage tatsächlich so in der Hand hielten, daß diese nach Osten und die andere nach. Westen zeigte. Nur im Bereich des „Sonnenaufgangs“ erwies sich eine „Verschüttung“ der Emmerähre als übles Zeichen. Im „Sonnenuntergang“" galt sie indes als günstiger Befund. Hieraus können wir schließen, daß - ganz so wie in den Eingeweideschauverfahren - auch in der Mehldivination die Lehre von ,rechten“ und ,linken“ Feldern bzw. von der pars familiaris und der pars hostilis ${ }^{14}$ eine entscheidende Rolle spielte. Denn der „Sonnenaufgang“ genannte Bereich ist nichts anderes als das

$13 \mathrm{Zu}$ positiv und negativ konnotierten Markierungen auf Leber und Eingeweiden siehe Meyer 1987 passim; Jeyes 1989, 81-96; Starr 1990, 1-lv; Leiderer 1990 passim; Koch-Westenholz 2000, 43-70. In Koch-Westenholz 2000, 41-42 sind die Wertigkeiten der Organmarkierungen in einer Tabelle zusammengestellt.

14 Siehe Starr 1983, 15ff. Die auch für die etruskische Eingeweideschau gültige Regel (siehe Thulin 1968, Band II, 26ff.) ist in einem keilschriftlichen Handbuch der Eingeweideschau formuliert: „Der Streifen zur Rechten (eines der zwölf konstitutiven Lebermerkmale oder eines anderen Eingeweidemerkmals) gehört mir an, doch der zur Linken dem Feind“ (Koch-Westenholz 2005, 114, Text Nr. 
,rechte' Feld, in dem sich Wohl und Wehe des Eigenen zeigte. Eine „Verschüttung" der Ähre verstand man hier als die „Verschüttung" der eigenen Person und damit als ein übles Zeichen für den Fragenden. Der „Sonnenuntergang“ hingegen erteilte als, linkes' Feld Auskunft über Erfolg und Mißgeschick des Widrigen. Eine „Verschüttung“ der Ähre galt hier als Hinweis auf widrige Bedingungen für das Widrige und geriet damit zum guten Zeichen. Selbstverständlich ist es kein Zufall, daß der "Sonnenuntergang“ mit dem Feindlichen und der Bereich der aufgehenden Sonne mit dem dem Menschen Zugewandten verbunden wurde. Denn seit alters her sah man in dem Ort des Sonnenuntergangs den Eingang in die Unterwelt, und deshalb wurde in Mesopotamien ebenso wie im Alten Ägypten der Westen mit dem Tod, der Osten hingegen mit Erneuerung und Leben assoziiert.

Es ist nicht auszuschließen, daß die Unterlage, auf die Mehl und Ähre, gewürfelt‘ wurden, so klein war, daß es kaum gelang, willentlich zu beeinflussen, in welches Feld die Ähre fallen würde. In diesem Fall hätte der Zufall darüber entschieden, ob ein Orakelbescheid mit Hilfe eines, linken' oder eines, rechten' Feldes zustande kam. Wahrscheinlich ist dies jedoch nicht. Denn mit Hilfe einer etwas größeren Unterlage, die sich wie eine Art Spielbrett in zwei Felder unterschiedlicher ,Ladung' teilt, hätte man leicht, ganz so wie dies in den Eingeweideschauen üblich war, ein zweistufiges Orakelverfahren durchführen und damit der kleinen, ,preisgünstigen' Opferschau ein Gepräge verleihen können, das die mit großem Aufwand betriebene Eingeweideschau kennzeichnete. In diesem wahrscheinlicheren Fall hätte man im Sonnenaufgangsfeld in einem ersten Durchgang das Ausmaß der günstigen Kräfte geprüft, und im Sonnenuntergangsfeld Informationen eingeholt, die über das Maß der Widrigkeiten Auskunft geben konnten, die dem Fragenden entgegenstehen würden.

Die Kenntnis der hier dargelegten, recht simplen Grundregeln der Mehldivination gestattet, die ratio nachzuvollziehen, die die hypothetischen Befunde der Omensammlung mit den ihnen zugeordneten Deutungen verbindet. Im folgenden gebe ich eine neue Übersetzung der ersten neun Einträge ${ }^{15}$ des von Jean Nougayrol edierten

3:44 (A i 59). Zu ,rechten` und ,linken` Feldern auf der Leber eines Opferlamms siehe auch Nougayrol 1968.

15 (1): Nougayrol 1963, 382:1-2; (2): ebd., 382:3-4; (3): ebd., 384:5-6; (4): ebd., 384:7-10; (5): ebd., 384:11-13; (6): ebd., 384:14-15; (7): ebd., 384:16-18; (4): 
altbabylonischen Schülermanuskriptes und füge jedem dieser neun Einträge eine kurze Begründung für die in der Handschrift gegebene Ausdeutung des Befundes bei:

(1) Wenn das Mehl nach „Sonnenuntergang“ hin die Emmer(ähre) [freigibt ${ }^{16}$ ]: Der kranke Mensch wird nicht am Leben bleiben.

Ein Befund im ,linken' Feld: Die frei daliegende Ähre zeigt hier gute Bedingungen für das Schlechte an. Ein ungünstiger Befund.

(2) Wenn das Mehl nach "Sonnenuntergang“ hin sich über die Emmer(ähre) türmt: Der kranke Mensch wird zwar lange krank sein, aber dann wird er leben.

Ein Befund im ,linken“ Feld: Die „Verschüttung“ der Ähre ist zwar hoch, aber sie tritt im ,linken' Feld auf und schützt so vor der Bedrohung des „Sonnenuntergangs“, der für Tod, Vernichtung, kurz für die stärkste negative Kraft steht. Der Fragende „wird leben“, aber wegen der Höhe der „Verschüttung“, die ihn dennoch trifft, zuvor „lange krank sein“. Ein günstiger Befund.

(3) Wenn das Mehl nach „Sonnenuntergang“ hin die Emmer(ähre) (in zwei Teile) zerspalten hat: Der betroffene Mensch wird rasch an seiner Krankheit sterben.

Ein Befund im ,linken' Feld: Die Ähre liegt nicht nur frei da, sie ist außerdem noch von einem dünnen Streifen Mehls in zwei Teile zerschnitten. Das ist zwar ausgesprochen günstig für das Schlechte, für den Fragenden aber ein denkbar ungünstiges Zeichen. Ein ganz und gar ungünstiger Befund.

(4) Wenn das Mehl nach „Sonnenuntergang“ hin auf seiner rechten und seiner linken Seite unversehrt geblieben ist, doch in seinem mittleren Bereich eingedellt: Um jenen Kranken wird man viel Geschrei machen, doch er wird gesund sein.

Ein Befund im ,linken' Feld: Die Ähre ist deshalb nicht erwähnt, weil sie in dem hier diskutierten Befund von dem Mehl vollständig bedeckt ist. Dies ist im, linken' Feld ein grundsätzlich gutes Zeichen. Lediglich im mittleren Bereich der „Verschüttung“ ist kein kleiner

ebd., 384:19-20; (5): ebd., 384:5.

16 Die hier vorgeschlagene Ergänzung wurde aufgrund inhaltlicher Erwägungen erschlossen. 
Gipfel aus Mehl, sondern eine Art Tal zu beobachten. Dies zeigt keine nachhaltigen Folgen an, sondern lediglich, daß man - freilich letztlich unberechtigt - ,viel Geschrei um den Kranken machen“ wird. Ein höchstens leicht eingeschränkt günstiger Befund.

(5) Wenn das Mehl nach „Sonnenuntergang “ $h i n$ im vorderen Bereich ${ }^{17}$ flach, im hinteren hoch aufgeschüttet daliegt: Jener Kranke wird leben, doch Dinge, die ihn ängstigen, werden in jenem Haus auftreten. ${ }^{18}$

Ein Befund im ,linken ' Feld: Eine vom Mehl verschüttete Emmerähre ist hier ein grundsätzlich gutes Zeichen. Dies wird ein wenig modifiziert durch den Umstand, daß die im , linken' Feld schützende „Verschüttung“ im ,vorderen Bereich“ recht flach war. Ein eingeschränkt günstiger Befund.

(6) Wenn das Mehl nach „Sonnenaufgang“ hin die Emmer(ähre) , auffrißt ${ }^{\circ 19}$ und dabei die linke Seite (des Mehlhäufchens) die rechte stützt: Der Todkranke wird sterben, doch das, was nach ihm kommt, wird gut bleiben.

Ein Befund im ,rechten“ Feld: Eine allseitige „Verschüttung“ der Ähre ist hier ein denkbar schlechtes Zeichen. Die Art der ,Verschüttung" läßt in allem Elend dennoch einen Hoffnungsschimmer erkennen. Der Omeneintrag zeigt, daß bei der Mehlwahrsagung die Befunde auf den ,rechten " und ,linken' Feldern ihrerseits jeweils in eine ,rechte" und ,linke" Seite eingeteilt wurden, welche für das „Eigene“ und das „Widrige“ stehen. Um den Schüler dies erkennen zu lassen, ist der Befund wohl in die didaktisch geschickt zusammengestellte Omensammlung aufgenommen: In einem ,rechten“ Feld „stützt" die linke Seite des Mehlhäufchens die rechte. Die linke Seite ist dabei die tragende Kraft, die rechte hingegen würde ohne die linke einbrechen. Die rechte Seite der „Verschüttung“ besitzt sozusagen keine eigene Energie. In einem

17 Der „vordere Bereich“ dürfte die dem Opferschauer zugewandte, nach Westen zeigende Seite der Unterlage sein. Denn vermutlich hielt der Opferschauer die "Sonnenaufgangsseite" der Unterlage, nach Osten schauend, der aufgehenden Sonne entgegen.

18 Die Lesung der Apodosis bleibt unsicher. Vielleicht ist: palhātūšsu ina bìtim šâti ibaššâ zu lesen. R. Pientka schlägt statt palhātūšsu die Lesung ahhātūušu („Schwestern“) vor. Dies scheint zwar epigraphisch möglich zu sein, ergibt aber keinen rechten Sinn.

19 Mehl, das die Ähre, auffrißt', dürfte wohl Mehl sein, das die Ähre von allen Seiten umhüllt. Anders als in Omen (2) liegt hier das Mehl auch unter der Ähre. 
positiv konnotierten Feld („Sonnenaufgang") besitzt die negativ konnotierte Markierung „Verschüttung“ im Bereich des „Eigenen“ (d.h. auf ihrer rechten Seite) einen Wert, der sich gewissermaßen als ,passiv ${ }^{6}$ erweist und nach Erfüllung des sich ankündigen Negativen keine Schadkraft mehr entfalten kann. Ein ganz und gar ungünstiger Befund mit einem kleinen Hoffnungsschimmer.

(7) Wenn das Mehl nach „Sonnenaufgang“ hin gleichmäßig die Emmer(ähre) , auffrißt', doch deren hinterer Teil (von dem Mehl nur) umgeben ist: Die Tage des Kranken werden sich nicht in die Länge ziehen, rasch wird er wieder am Leben sein.

Ein Befund im ,rechten F Feld: Die Ähre ist nur teilweise „verschüttet", ihr hinterer Teil, also der nach Osten zeigende Teil (vgl. Anm. 17) ist frei von Mehl. Der Befund wird entsprechend dem Sonnenlauf von „Sonnenaufgang“ nach „Sonnenuntergang“ gelesen. Der hintere Teil der Ähre ist so der aussagekräftigere. Da er freiliegt, wird der Mensch bald ,wieder am Leben sein“. Aus dem mit Mehl verdeckten Teil der Ähre liest man die derzeit bestehende „Krankheit‘. Der Befund vermittelt dem Schüler, der sich durch das Studium der Omeneinträge den Regeln des Verfahrens studierend und verstehend nähern soll, daß die positive ,Ladung' des ,rechten Feldes von Innen nach Außen aus dem Blick des Zeichendeuters bzw. von West nach Ost zunimmt. ${ }^{20}$ Ein günstiger Befund.

(8) Wenn das Mehl nach „Sonnenaufgang “ hin (in einer dünnen Schicht) zerstäubt daliegt: Den betroffenen Menschen hält ein Totengeist gepackt; jener Totengeist ist die „Hand seines Vaters“.

Ein Befund im ,rechten` Feld: Die Ähre ist nicht erwähnt und deshalb als vom Mehl „verschüttet" anzusehen. Während bislang von hohen "Verschüttungen“ die Rede war, wird hier der Fall eingeführt, daß die Ähre nur mit einer hauchdünnen Schicht des Mehls bestäubt ist. In einem, rechten' Feld ist dieser Befund grundsätzlich negativ. Er wird freilich durch den geringfügigen Grad der „Verschüttung“ stark relativiert. Ein bedrohlicher, aber letztlich unentschiedener Befund mit einer negativen Tendenz.

(9) Wenn das Mehl nach „Sonnenaufgang" hin wie das Gesicht eines Löwen ausschaut: Den betroffenen Menschen hält ein Totengeist gepackt, der in der Steppe daliegt; die Sonne wird um seinetwillen

20 Entsprechend nimmt die negative Ladung eines, linken' Feldes von Innen nach Außen aus dem Blick des Zeichendeuters bzw. von Ost nach West zu. Vergleichbares ist auch aus der Eingeweideschau bekannt. 
(d.h. um des Menschen willen) zum dem (alles) verwehenden Wind schicken, so daß er am Leben bleibt.

Ein Befund im, rechten'Feld: Hier geht es um einen Befund, der sich von dem vorangehenden nur dadurch unterscheidet, daß die dünne Mehlschicht, die die Emmerähre überzieht, ein bestimmte Gestalt angenommen hat, die - so wohl das didaktische Erkenntnisziel der Omensammlung - dem Befund eine eigene Konnotation gibt. Die Form des Löwengesichts, welche die Mehlschicht annahm, ist (aus Gründen, die wir noch nicht wirklich durchschauen) eine positive Markierung, welche Einfluß auf die Wertung des Gesamtbefundes hat. Ein bedrohlicher, aber letztlich unentschiedener Befund mit einer positiven Tendenz.

Die Besprechung der Omeneinträge dürfte gezeigt haben, mit welch großem didaktischen Geschick diese neun Fälle ausgewählt wurden. Denn mit einigem Nachdenken ermöglicht das Studium der paradigmatischen Fälle, die hermeneutischen Regeln des Divinationsverfahrens zu erkennen. Es weckt auch die Sensibilität dafür, daß die ermittelten positiven und negativen Befunde ihrerseits günstige oder ungünstige Tendenzen zu erkennen geben.

Die Deutungen der Omenbefunde handeln von Krankheit und Genesungschancen, wohl nicht zuletzt, weil das Mehlorakel vermutlich tatsächlich oft befragt wurde, um Einblicke in das Schicksal eines leidenden Angehörigen zu erhalten. Die vielleicht nur scheinbare thematische Eingrenzung der Ausdeutungen sollte indes nicht überschätzt werden. Denn das sich darin spiegelnde Ringen von Bedrohung und Heil läßt sich leicht auf alle nur denkbaren Lebensbereiche und Umstände übertragen. In diesem Sinn kann es ohne weiteres als Allegorie auf das Ringen von dem Widrigen mit dem Günstigen verstanden werden. Der thematisch gebundenen Formulierung der Apodosen zum Trotz generierte man mit der Mehlwahrsagung ja stets nur ein Zeichen göttlicher Gunst oder Zurückweisung. Aus diesem Grund konnte die Aleuromantie, so wie auch die Eingeweideschau, grundsätzlich zur Beantwortung jeder beliebigen Entscheidungsfrage genutzt werden.

In den letzten fünf Omeneinträgen unserer altbabylonischen Schülertafel - und nur dort - ist eine weitere Methode dokumentiert, 
um mit Hilfe von Opfermehl einen Orakelbescheid $\mathrm{zu}$ erstellen. ${ }^{21}$ Dennoch gelingt es mit den wenigen dort zu findenden Angaben nicht einmal, nur die wesentlichen Züge des Verfahrens klar zu erkennen. Es scheint so, als habe man den oben beschriebenen Vorgang des ,Auswürfelns' von Mehl, unter das man eine Emmerähre gemischt hatte ${ }^{22}$ siebenmal wiederholt, um dann die Serie von sieben Würfen insgesamt zu beurteilen. Die einzelnen Mehlhäufchen teilte man dabei in ,günstige", die als „die der rechten Seite ${ }^{\text {“23 }}$ bezeichnet wurden, und in , ungünstige" ein.

In einer aus dem neuassyrischen Ninive bekannten Edition der šumma ālu genannten Sammlung terrestrischer Omina findet sich ein kleiner Abschnitt, der auf die Existenz eines dritten Verfahrens der Mehldivination weist. ${ }^{24}$ Dabei wurde Mehl in eine mit Wasser gefüllte Schale gestreut und beobachtet, wie sich das Mehl auf dem Wasser verhielt. Teilte es sich auf der Wasseroberfläche schwimmend in zwei Inseln, so galt dies als Zeichen dafür, daß „,der Gott das Opfer des Menschen angenommen" hatte, wohl da sich das für den Menschen stehende Mehl geteilt hatte, als auch der Opferspender seinen Besitz mit der Gottheit teilen wollte. Blieb das Mehl zusammenhängend, sah man darin ein Zeichen des Gotteszorns. Dieses simplen Verfahrens bediente man sich wohl tatsächlich, um die Annahme eines Opfers zu überprüfen. Darüber hinaus konnte man mit dieser Methode auch eine positive oder negative Antwort auf jede beliebige Entscheidungsfrage erzielen. Ging nämlich das Mehl im Wasser unter, so würde auch der Fragende untergehen, tauchte es wieder auf, so sah man darin einen Hinweis darauf, daß der Fragende letztlich aus seinem Elend wieder herausfinden würde. Wenn sich das Mehl in einer dünnen Schicht auf der gesamten Wasseroberfläche ausbreitete, galt das als ein unklares Zeichen. Ein übles hingegen war es, wenn sich das Mehl zusammenballte.

21 Nougayrol 1963, 384:25-35.

22 Schon dies bleibt sehr unsicher.

23 Vgl. Nougayrol 1963, 384:25.

24 Die Passage aus der Omenserie šumma ālu findet sich in: Nötscher 1930, 204 $207=$ Boissier $1905,173-175=$ CT 39, 36, K 4097+:80-92 und in einer noch unveröffentlichten Parallelstelle: K 57, Rs. 4 -9 (zu K 57 siehe Nougayrol 1967, 35-37). 
In der keilschriftlichen Omenliteratur ist dieses dritte Verfahren der Mehldivination allein in der hier zitierten Omenpassage bezeugt. Es wäre indes ganz falsch, daraus zu folgern, daß es von nur geringer Bedeutung war. Denn die Existenz unseres Verfahrens läßt sich nicht nur in der im 7. Jh. v. Chr. niedergeschriebenen Omenpassage nachweisen, sondern auch in einer 1500 Jahre älteren sumerischen Königsinschrift, und es ist daher nicht unwahrscheinlich, daß man diese dritte Form der Mehldivination seit dem dritten vorchristlichen Jahrtausend durchgängig über viele Jahrhunderte hinweg praktizierte. Außerdem wurde das mit Mehl und Wasser ausgeführte Orakel keineswegs - so wie die schon besprochenen Verfahren der Mehlwahrsagung ausschließlich von ärmeren Leuten in Anspruch genommen. Auch in Riten und Ritualen der Mächtigen hatte es seinen Platz. Der mehr als 4000 Jahre alten sumerischen Königsinschrift können wir nämlich entnehmen, daß Gudea, Stadtfürst von Lagasch, den Bau eines neuen Tempels nicht begann, ohne sich hierfür mit divinatorischen Mitteln gleich dreifach der Zustimmung seines Gottes zu versichern, obgleich doch der Bauplatz schon vorbereitet und alles benötigte Material längst zur Hand war. Nach einer günstig verlaufenen Eingeweideschau vergewisserte sich der Fürst des Wohlwollens seines Gottes, indem er „Getreide auf frisches Wasser gab“, um schließlich die dritte göttliche Zusage in einem Traumgesicht zu erhalten, das sich dem Herrscher im Rahmen eines Inkubationsrituals offenbarte. ${ }^{25}$

Ganz in der Weise, wie in der šumma ālu-Passage beschrieben, ist das von Gudea erwähnte Verfahren der Mehldivination sogar dem Dichter der Odyssee als eine Methode geläufig, um rasch zu prüfen, ob ein Opfer bei den Göttern Gnade finde. ${ }^{26}$ Daraus darf man wohl schließen, daß in jener Zeit das zuletzt beschriebene Verfahren der Aleuromantie im gesamten alten Vorderasien Verbreitung gefunden hatte.

In der griechisch-römischen Welt blieb die zumeist Aleuromantie (,Weizenwahrsagung“) oder Alphitomantie („Gerstenwahrsagung“) genannte Mehldivination bis in die Spätantike bekannt. So wie im Alten Orient der Sonnengott Schamasch, galt - dem spätantiken

25 Siehe Suter 2000, 92 mit Anm. 103 zu Gudeas ,Zylinder A“, Kol. 20:5-12. Mit dem Mehlorakel wurde wohl bestätigt, daß für das Traumorakel der rechte Zeitpunkt gekommen war (hierzu vgl. George/al-Rawi 1996, 173f.).

26 Siehe Odyssee III:440ff. 
Lexikographen Hesychios zufolge - nun Apollon als aleuromantis, also als der Gott, der den Menschen durch mit Mehl hervorgebrachte Zeichen Leitung zu geben versprach. ${ }^{27}$ Erst die Christen sahen in den Formen der Mehlwahrsagung, wie der byzantinische Mönch Georgios Monachos in seiner Weltchronik noch im 9. Jh. unserer Zeitrechnung ausführte, ${ }^{28}$,teuflische Prophetie“, die es zu bekämpfen galt.

\section{Bibliographie}

Abbink, J.

1993: Reading the entrails: analysis of an African divination discourse, Man. New Series, Vol. 28, 705-726.

Archi, A.

1975: L'ornitomanzia ittita, SMEA 16, 119-180.

Blome, $\mathrm{F}$.

1934: Die Opfermaterie in Babylonien und Israel, I. Teil, Sacra scriptura antiquitatibus orientalibus illustrata 4 , Romae.

Boissier, A.

1905: Choix de textes relatifs à la divination assyro-babylonienne, Genève.

Butler, S.A.L.

1998: Mesopotamian Conceptions of Dreams and Dream Rituals, AOAT 258, Münster.

de Boor, C.

1978: Georgii Monachi chronicon. Band 1: Textum genuinum usque ad Vespasiani imperium continens; Band 2: Textum genuinum inde a Vespasiani imperio continens, Leipzig 1904.

Durand, J.-M.

1988: Archives épistolaires de Mari I/1 (Archives royales de Mari 26/1), Paris.

1997: La divination par les oiseaux, MARI 8, 273-282.

27 Ganschinietz 1918, 78.

28 De Boor 1978, Band 1, 237:20-239, 15. 
Edzard, D. O.

2004: Altbabylonische Literatur und Religion, in: D. Charpin/D. O. Edzard/ M. Stol (Hrsg.), Mesopotamien. Die altbabylonische Zeit, OBO 160/4, Fribourg/Göttingen, 481-640.

Ganschinietz, R.

1918: Aleuromanteía, in: Paulys Real-Encyclopädie der classischen Altertumswissenschaft, Suppl. 3, 78-79.

George, A. R./al-Rawi, F. N. H.

1996: Tablets from the Sippar Library VI. Atra-ḩasīs, Iraq 58, 147-190.

Haas, V.

2008: Hethitische Orakel, Vorzeichen und Abwehrstrategien, Berlin.

Jeyes, U.

1989: Old Babylonian Extsipicy, PIHANS 64, Leiden.

Koch-Westenholz, U.

2000: Babylonian Liver Omens. The Chapters Manzāzu, Padānu and Pān tākalti of the Babylonian Extispicy Series mainly from Aššurbanipal's Library, Copenhagen.

2005: Secrets of Extispicy. The Chapter Multābiltu of the Babylonian Extispicy Series and Nișirti barûti Texts mainly from Aššurbanipal's Library, AOAT 326, Münster.

Leiderer, R.

1990: Anatomie der Schafsleber im babylonischen Leberorakel, München/ Bern/Wien/San Francisco.

Maul, S. M.

1994: Zukunftsbewältigung. Eine Untersuchung altorientalischen Denkens anhand der babylonisch-assyrischen Löserituale (Namburbi), BF 18, Mainz.

2003: Omina und Orakel. A. In Mesopotamien, in: D. O. Edzard (Hrsg.), RIA, Band 10, 1./2. Lieferung Oannes - Pabilsag(a), Berlin/New York, 45-88.

2007: Ringen um göttliches und menschliches Maß. Die Sintflut und ihre Bedeutung im Alten Orient, in: E. Hornung/A. Schweizer (Hrsg.), Schönheit und Maß. Beiträge der Eranos Tagungen 2005 und 2006, Basel, 161-183.

2008: Den Gott ernähren. Überlegungen zum regelmäßigen Opfer in altorientalischen Tempeln, in: E. Stavrianopoulou, A. Michaels/ C. Ambos (Hrsg.), Transformations in Sacrificial Practices. From Antiquity to Modern Times, Berlin, 75-86. 
Meyer, J.-W.

1987: Untersuchungen zu den Tonlebermodellen aus dem Alten Orient, AOAT 39, Kevelaer/Neukirchen-Vluyn.

Nötscher, F.

1930: Die Omen-Serie šumma âlu ina mêlê šakin (CT 38-40) (Fortsetzung), Or 51-54.

Nougayrol, J.

1963: Aleuromancie babylonienne, OrNS 32, 381-386.

1967: „Oiseau“ ou oiseau?, RA 61, 23-38.

1968: Le foie „d'orientation“ BM 50494, RA 62, 31-50.

Pientka-Hinz, R.

2008: 1. Omina und Prophetien, in: B. Janowski, G. Wilhelm (Hrsg.), Omina, Orakel, Rituale und Beschwörungen, TUAT NF 4, 16-60.

Schramm, W.

2008: Ein Compendium sumerisch-akkadischer Beschwörungen, Göttinger Beiträge zum Alten Orient 2, Göttingen.

Starr, I.

1983: The Rituals of the Diviner, BiMes 12.

1990: Queries to the Sun God. Divination and Politics in Sargonid Assyria, SAA 4, Helsinki.

Suter, C. E.

2000: Gudea's Temple Building. The Representation of an Early Mesopotamian Ruler in Text and Image, CM 17, Groningen.

Thulin, C. O.

1968: Die etruskische Disziplin, Darmstadt [unveränderter reprografischer Nachdruck aus: Göteborgs Högskolas Årskrift, Band XI (1905): 5, Band XII (1906): 1, Band XV (1909): 1].

Tsukimoto, A.

1982: A Report on Divination by Means of a Sacrificial Bird, Orient 18, $107-110$.

Zimmern, $\mathrm{H}$.

1901: Beiträge zur Kenntnis der babylonischen Religion, $A B$ 12, Leipzig 1896 und 1901. 